\title{
Frequency of Macroprolactinemia in Hyperprolactinemic Women Presenting with Menstrual Irregularities, Galactorrhea, and/or Infertility: Etiology and Clinical Manifestations
}

\author{
Alfredo Leaños-Miranda, ${ }^{1}$ Karla Leticia Ramírez-Valenzuela, ${ }^{1}$ Inova Campos-Galicia, \\ Rosario Chang-Verdugo, ${ }^{2}$ and Lizbeth Zarela Chinolla-Arellano ${ }^{1}$ \\ ${ }^{1}$ Research Unit in Reproductive Medicine, Unidad Médica de Alta Especialidad, Hospital de Ginecología y Obstetricia, \\ Luis Castelazo Ayala, Instituto Mexicano del Seguro Social, Don Luis No. 111, Col. Nativitas, 03500 México, DF, Mexico \\ ${ }^{2}$ Clinical Laboratory, Unidad Médica de Alta Especialidad, Hospital de Ginecología y Obstetricia, Luis Castelazo Ayala, \\ Instituto Mexicano del Seguro Social, México, DF, Mexico
}

Correspondence should be addressed to Alfredo Leaños-Miranda; alfredolm@yahoo.com

Received 1 May 2013; Revised 15 August 2013; Accepted 30 August 2013

Academic Editor: Mario Maggi

Copyright (C) 2013 Alfredo Leaños-Miranda et al. This is an open access article distributed under the Creative Commons Attribution License, which permits unrestricted use, distribution, and reproduction in any medium, provided the original work is properly cited.

\begin{abstract}
Aim. To determine the frequency of macroprolactinemia, its etiology, and the clinical manifestations in patients with hyperprolactinemia presenting with menstrual irregularities, galactorrhea, and/or infertility who were attended by the gynecologyendocrinology service. Methods. In a cross-sectional study, 326 hyperprolactinemic women were tested for serum prolactin (PRL) concentrations before and after chromatographic separation (gel filtration and affinity with protein G) and extraction of free PRL with polyethylene glycol (PEG). Results. Sera from 57 patients (17.5\%) were found to have macroprolactinemia. The presence of macroprolactinemia was attributable to anti-PRL autoantibodies in 54 (94.7\%) patients. The median serum PRL levels were similar in patients with or without macroprolactinemia $(42.0$ versus $38.1 \mathrm{ng} / \mathrm{mL})$. In contrast, patients with macroprolactinemia had lower serum-free PRL levels (median 9.2 versus $31.7 \mathrm{ng} / \mathrm{mL}, P<0.001$ ). Patients without macroprolactinemia had a higher frequency of galactorrhea and abnormal pituitary imagine findings $(P<0.002)$. Conclusions. We can conclude that macroprolactinemia should be considered as a benign variant, and it must be ruled out in women presenting with menstrual irregularities, galactorrhea, and/or infertility in order to investigate other causes different than hyperprolactinemia. Serum PRL precipitated with PEG is a convenient and simple procedure to screen for the presence of macroprolactinemia.
\end{abstract}

\section{Introduction}

Prolactin (PRL) is a polypeptide hormone primarily secreted by the anterior pituitary gland.

The presence of several PRL isoforms in serum and other biological fluids has been clearly established. The major circulating isoform of PRL is a $23 \mathrm{kDa}$ single polypeptide chain (monomeric PRL), which comprises up to $80 \%$ of the total PRL in serum from normal subjects and the majority of patients with hyperprolactinemia (HPRL). In addition, there are two other PRL isoforms that display higher molecular weights, referred to as big PRL $(45-50 \mathrm{kDa})$ and big big PRL ( $>100 \mathrm{kDa})$ and also known as macroprolactin [1]. The presence of these isoforms has been attributed to formation of aggregates of monomeric PRL with different glycosylation degrees and binding of PRL to serum protein in circulation, mainly to anti-PRL autoantibody of IgG isotype [2-5]. These structural modifications may distinctly affect the biological and immunological properties of the hormone [5]. Immunometric methods, which are commonly used to determine serum PRL, are largely blind to changes in the patterns and proportions of PRL isoforms, which may influence both the net in vivo biological activity of the hormone and the clinical features of PRL-related disease states. To date, it is well recognized that the molecular heterogeneity of PRL is present in sera from hyperprolactinemic subjects. Predominant presence of big big PRL, a phenomenon termed macroprolactinemia (MPRL), has been reported in 15 to $46 \%$ of subjects 
with HPRL [6-13]. Although the nature of MPRL is still unclear, much evidence indicates that big big PRL is mostly an IgG-23 kDa PRL complex (i.e., anti-PRL autoantibodymonomeric PRL) $[3,14,15]$. Macroprolactin is big enough to be confined to vascular spaces, and therefore HPRL develops due to slower serum clearance of macroprolactin [16] and also due to lack of negative feedback, because macroprolactin cannot freely access the hypothalamus [14]. In addition, macroprolactin displays low biological activity in vitro $[5,17]$. Independently of the nature of big big PRL (i.e., due or not to anti-PRL autoantibodies), clinical symptoms of HPRL, such as amenorrhea and galactorrhea in women and impotence in men, are usually less frequent or even absent in patients with MPRL $[2,4,18]$. However, several patients with MPRL cannot be distinguished from true hyperprolactinemic patients on the basis of clinical features alone [11, 12, 19].

Since signs and symptoms of HPRL are nonspecific and relatively common, it is possible that some patients with MPRL experience these signs and symptoms coincidentally, but unrelated to PRL, resulting in misdiagnosis and inappropriate treatment [20].

Since there is scarce information on the frequency of MPRL in hyperprolactinemic women with signs and symptoms related to hyperprolactinemia, the aim of the present work was to study MPRL frequency and its etiology in hyperprolactinemic women presenting with menstrual irregularities, galactorrhea, infertility, or/and alterations in libido who were attended by the gynecology and endocrinology service of a third level care hospital, as well as to determine the utility of the percentage of serum PRL precipitated with polyethylene glycol (PEG) assay in the detection of MPRL and to establish the ideal cut-off point for this test.

\section{Material and Methods}

The study protocol was approved by the Human Ethical Committee and Medical Research Council of the Instituto Mexicano del Seguro Social. Written informed consents were obtained from all study participants.

All participant women were patients attended by the Gyneco-Endocrinology outpatient clinic of our hospital; those with serum PRL levels greater than $25 \mathrm{ng} / \mathrm{mL}$ were included in the study. A clinical history was obtained with special reference to the presence of menstrual irregularities, galactorrhea, infertility, or alterations in libido. Between six and twelve months later, from clinical chart, we obtained information on imaging investigations, diagnoses, and treatments used.

A venous blood sample was drawn between 07:00 and 08:00 a.m., under basal conditions and without hormonal or drug stimulus. Sera were stored at $-70^{\circ} \mathrm{C}$ until being used.

2.1. Determination of Direct or Total Serum PRL Levels. PRL concentration in serum was measured by an ultrasensitive enzyme immunoassay previously described [21]. This particular method has shown a high reliability for detecting serum PRL independently of its isoform composition; in this assay the presence of anti-PRL autoantibodies does not interfere with the test results, yielding concentration values of 5$25 \mathrm{ng} / \mathrm{mL}$ in normal conditions. The sensitivity of the assay was $0.018 \mathrm{ng} / \mathrm{mL}$, and the withinassay and between assay coefficients of variation were $5.7 \%$ and $6.8 \%$, respectively.

2.2. Determination of Serum Free or Monomeric PRL Levels. Free PRL was extracted from the serum using PEG, as previously described [22].

2.3. Gel Filtration Chromatography and Affinity Chromatography. Gel filtration was performed on Sephacryl $200 \mathrm{HR}$ column $(60 \times 1 \mathrm{~cm})$ (GE Biosciences), using a previously described procedure [22]; recovery of PRL was $110 \pm 8.4 \%$, on average. Affinity chromatography for IgG was performed using $1 \mathrm{~mL}$ protein-G Sepharose columns (Gammabind G, GE-Healthcare, Chalfont St. Giles, UK), as described [14, 22]. Immunoreactive PRL present in eluate fractions was determined by the same ultrasensitive enzyme immunoassay. Three patterns were observed in gel filtration chromatography, determined by analysis of the area under the PRL elution curve: (1) exclusive or predominant pattern of MPRL ( $\geq 50 \%$ of big big PRL), (2) exclusive or predominant pattern of monomeric PRL ( $\geq 50 \%$ of little PRL), and (3) variable pattern, without a predominant pattern of the previously mentioned possibilities.

In addition, serum samples were considered to contain anti-PRL autoantibodies when the retained percentage of PRL by the protein-G Sepharose column was above $4.2 \%$ this value represents the mean +3 SD obtained from sera of 40 healthy pregnant women without MPRL and who had little PRL as the predominant circulating species of PRL ( $\geq 95 \%$ as confirmed by size exclusion chromatography).

2.4. Statistical Analysis. The significance of differences between continuous variables was determined by the nonpaired Student's $t$-test (or Mann-Whitney $U$ test for nonnormally distributed variables). Differences between categorical variables were determined by the Chi-square test with Yates's continuity correction (or Fisher's exact test for small samples). The lineal relationship between serum total PRL levels and the percentage of bound PRL-IgG (anti-PRL autoantibody), as well as between relative amounts of big big PRL determined by gel filtration chromatography and the percentage of PRL precipitated with PEG was assessed by the Pearson correlation coefficient. A receiver operating characteristic curve for the percentage of serum PRL precipitated with PEG assay was generated. The area under the curve was calculated to establish the ideal cut-off point for this test for the detection of MPRL. A 2-tailed $P<0.05$ was considered statistically significant.

\section{Results}

3.1. General Description. The study sample consisted of 326 hyperprolactinemic patients. Mean age was $31.4 \pm 2.9$ years (range 20-42). Indications for testing serum PRL levels include the following: menstrual irregularities, galactorrhea, infertility, and/or alterations in libido (Table 1). Median 
TABLE 1: Demographic and clinical data, serum total and free immunoreactive prolactin (PRL) levels, distribution of PRL immunoreactivity in three fractions obtained after gel filtration, and percentage of retained PRL in affinity chromatography in hyperprolactinemic patients according to the absence or presence of macroprolactinemia (MPRL).

\begin{tabular}{|c|c|c|c|}
\hline Variable & Without MPRL $(n=269)$ & With MPRL $(n=57)$ & $P$ value \\
\hline Age, yrs, mean \pm SD & $33.6 \pm 8.9$ & $32.9 \pm 9.1$ & $0.62^{\mathrm{b}}$ \\
\hline Body mass index, mean $\pm \mathrm{SD}$ & $27.4 \pm 4.8$ & $27.7 \pm 4.1$ & $0.72^{\mathrm{b}}$ \\
\hline Direct PRL, ng/mL, median (range) & $38.1(25.5-1,860.0)$ & $42.0(26.0-268.6)$ & $0.27^{\mathcal{C}}$ \\
\hline Free PRL, ng/mL, median (range) & $31.7(25.5-1,840)$ & $9.2(2.2-22.2)$ & $<0.001^{\mathrm{c}}$ \\
\hline Big big PRL (\%), mean \pm SD & $2.9 \pm 1.2$ & $72.0 \pm 11.2$ & $<0.001^{\mathrm{b}}$ \\
\hline Big PRL (\%), mean \pm SD & $2.5 \pm 2.4$ & $3.1 \pm 2.1$ & $0.85^{\mathrm{b}}$ \\
\hline Little PRL (\%), mean \pm SD & $96.0 \pm 8.7$ & $24.9 \pm 10.1$ & $<0.001^{\mathrm{b}}$ \\
\hline IgG-bound PRL (\%), mean $\pm S^{a}$ & $0.8 \pm 1.4$ & $38.5 \pm 4.5$ & $<0.001^{\mathrm{b}}$ \\
\hline Anti-PRL autoantibodies (\%) & $0(0)$ & $54(94.7)$ & $<0.001^{\mathrm{d}}$ \\
\hline Oligomenorrhea or amenorrhea (\%) & $116(44.8)$ & $28(49.1)$ & $0.50^{\mathrm{d}}$ \\
\hline Galactorrhea (\%) & $91(33.8)$ & $7(12.3)$ & $0.002^{\mathrm{d}}$ \\
\hline Infertility (\%) & $85(31.6)$ & $21(36.8)$ & $0.54^{\mathrm{d}}$ \\
\hline Alterations in libido (\%) & $13(4.8)$ & $1(1.8)$ & $0.50^{\mathrm{e}}$ \\
\hline Secondary hyperprolactinemia (\%) & $81(30.1)$ & $6(10.5 \%)$ & $0.004^{\mathrm{d}}$ \\
\hline Polycystic ovarian syndrome (\%) & $22(8.2)$ & $4(7.0)$ & $0.93^{\mathrm{e}}$ \\
\hline Dopamine agonist prescribed (\%) & $147(54.6)$ & $44(77.2)$ & $0.003^{\mathrm{d}}$ \\
\hline
\end{tabular}

${ }^{a}$ PRL retained in protein G-Sepharose column (PRL retained/PRL nonretained + PRL retained $\left.\times 100 \%\right)$.

${ }^{\mathrm{b}}$ Nonpaired Student's $t$-test, ${ }^{\mathrm{c}}$ Mann-Whitney $U$-test, ${ }^{\mathrm{d}} \chi^{2}$ test, and ${ }^{\mathrm{e}}$ Fisher's exact test.

serum PRL was $38.3 \mathrm{ng} / \mathrm{mL}$ (range 25.5-1,860.0 ng/mL). Among of these patients with HPRL, in 87 (26.7\%) there was an identifiable condition that could account for the increased PRL levels (secondary HPRL): 61 patients were attributable to overt or subclinical hypothyroidism, evaluated on the basis of normalization of TSH and PRL concentrations with L-thyroxine, except in six patients who had MPRL and remained hyperprolactinemic despite the normalization of TSH levels; 22 patients were attributable to prolactinomas, and 4 patients were attributable to the use of medications. In the remaining 239 patients $(73.3 \%)$, no cause could be identified that would explain the presence of HPRL (idiopathic HPRL).

3.2. Frequency of MPRL and Its Etiology. According to gel filtration profiles of immunoreactive PRL, sera from 57 hyperprolactinemic patients were found to have an exclusive or predominant pattern of MPRL (17.5\%; confidence interval (CI), 95\%, 13.4-21.6\%) (Table 1, Figure 1). The frequency of MPRL among patients with secondary HPRL (all with hypothyroidism) was $6.9 \%$ (95\% CI 1.6-12.2\%). In contrast, 51 of the 239 patients with idiopathic HPRL had MPRL (21.3\%, 95\% CI 16.1-26.5\%; $P=0.014)$.

Sera from two patients displayed a variable pattern $(0.6 \%)$ with a percentage of MPRL of 40.8 and $44.6 \%$, respectively, and the remaining 267 patients $(81.9 \%)$ had an exclusive or predominant pattern of monomeric PRL (23 kDa, Figure 1).

In 54 of 57 patients with MPRL, a significant amount of immunoreactive PRL was retained on the protein Gsepharose column $(42.5 \pm 3.9 \%$ versus $0.8 \pm 1.4 \%(P<$ 0.001 ) in sera without MPRL) (Figure 2). In the 3 remaining sera with MPRL, almost all immunoreactive PRL passed through the protein G-sepharose column, and it was similar in sera without MPRL. Then, the frequency of anti-PRL autoantibodies was $94.7 \%$ (95\% CI 88.9-100\%). Data also showed a positive correlation $(r=0.63, P<0.001)$ between the percentage of serum PRL retained on protein G-sepharose column and serum PRL levels.

3.3. Characterization of Patients with MPRL. To characterize hyperprolactinemic patients with and without MPRL, a comparison was made among their clinical and laboratory variables (Table 1). We found that the median of total PRL levels in patients with MPRL was not different from that of patients without MPRL (42.0 ng/mL (range 26.0-268.6) versus $38.1 \mathrm{ng} / \mathrm{mL}$ (range 25.5-1,860.0), $P=0.27$ ); in contrast, the median of free PRL levels was significantly lower $(9.2 \mathrm{ng} / \mathrm{mL}$ (range 2.2-22.2) versus $31.7 \mathrm{ng} / \mathrm{mL}$ (range 25.5-1,840.0) $P<0.001)$. Gel filtration chromatography profiles of immunoreactive PRL in sera from patients with MPRL showed that much of $(72.0 \pm 10.0 \%)$ PRL was eluted as big big PRL (approximately $150 \mathrm{kDa}$ ). By contrast, in patients without MPRL, the $23 \mathrm{kDa}$ form of PRL remained the predominant species identified $(96.0 \pm 8.7 \%)$. Likewise, when serum IgG-bound PRL was extracted by affinity chromatography, a significant amount $(38.5 \pm 4.5 \%)$ of immunoreactive PRL was coeluted with IgG fractions in samples from patients with MPRL, while marginal amounts of PRL were detected in $\mathrm{IgG}$ fractions from patients without MPRL $(0.8 \pm 1.4 \%$, $P<0.001)$.

On the other hand, the frequency of galactorrhea was significant higher in patients without MPRL than in those with MPRL $(P=0.002)$, whereas there was no difference in frequency of oligomenorrhea, amenorrhea, infertility, or alterations in libido between the two groups. Finally, six of the 57 patients with MPRL had secondary causes of HPRL 


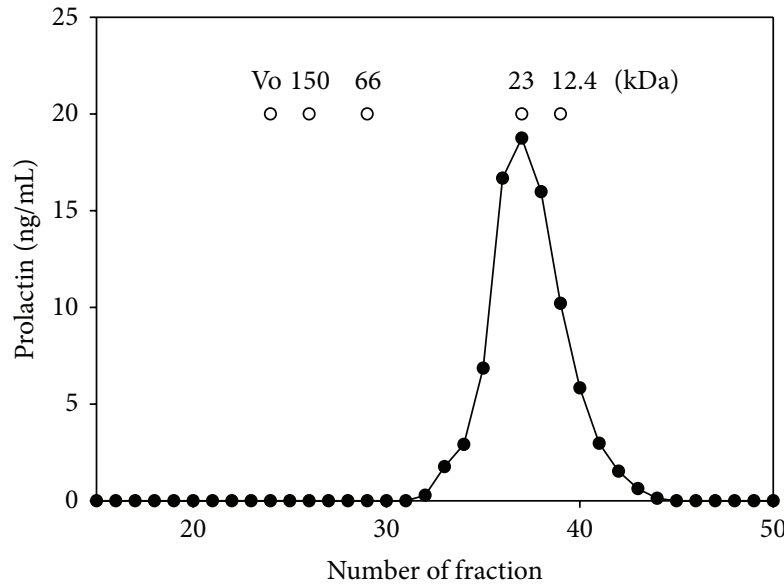

(a)

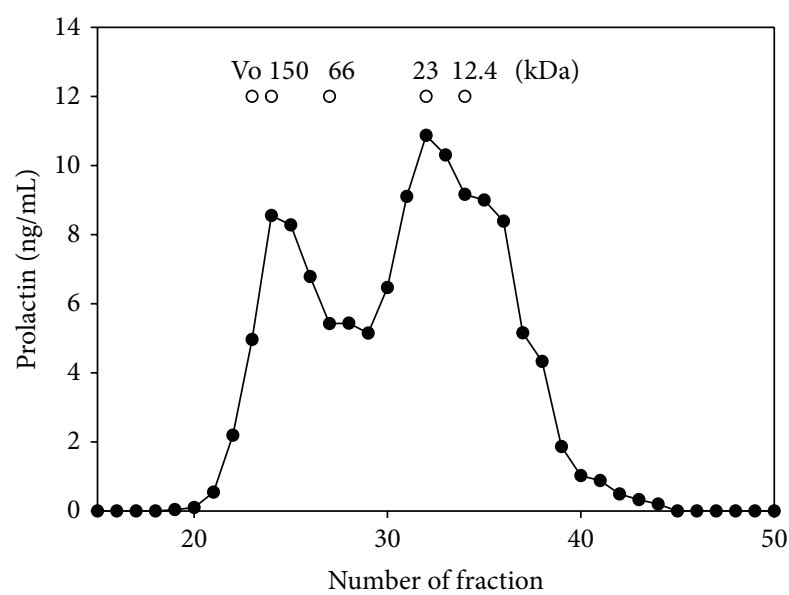

(c)

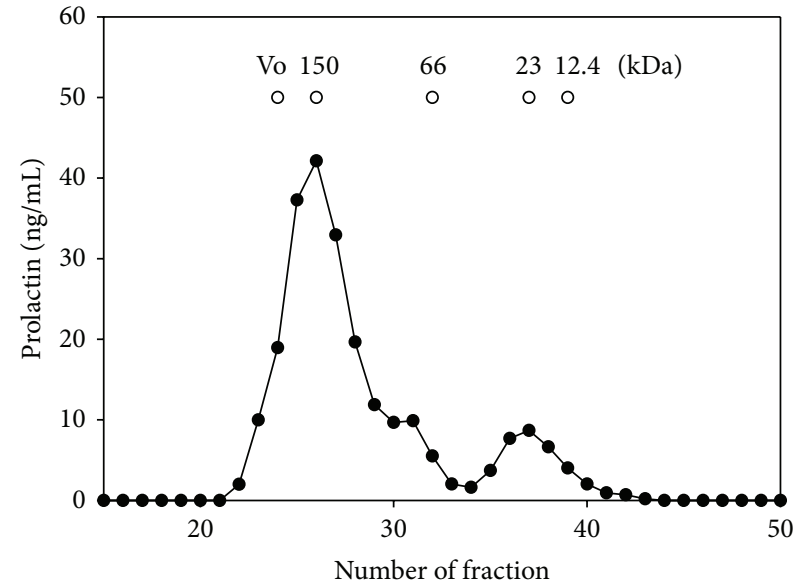

(b)

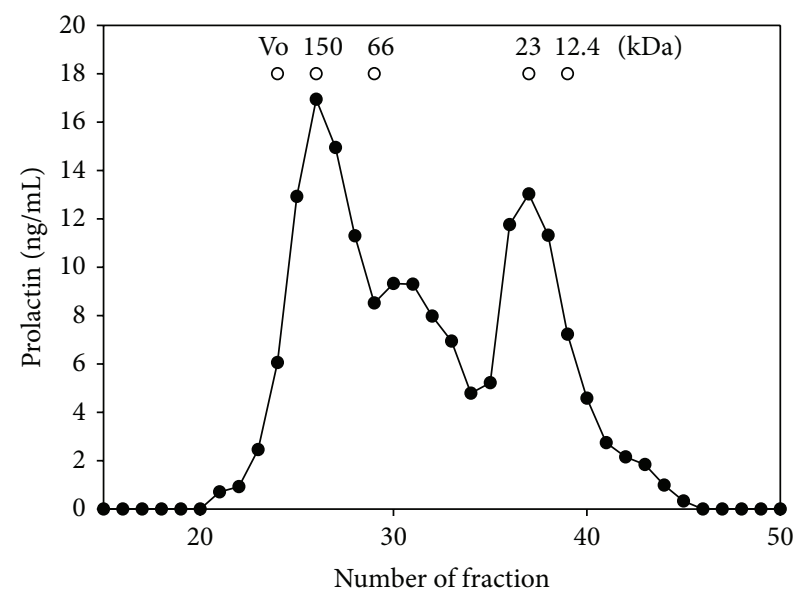

(d)

FIGURE 1: Representative gel filtration profiles of immunoreactive PRL in sera from hyperprolactinemic patients on a Sephacryl HR 200 column $(60 \times 1 \mathrm{~cm})$. Samples $(1 \mathrm{~mL})$ were applied on the column, and the fractions of $900 \mu \mathrm{L}$ were collected. (a) Exclusive or predominant pattern of monomeric PRL (little PRL); (b) exclusive or predominant pattern of macroprolactinemia (big big PRL); ((c) and (d)) variable patterns.

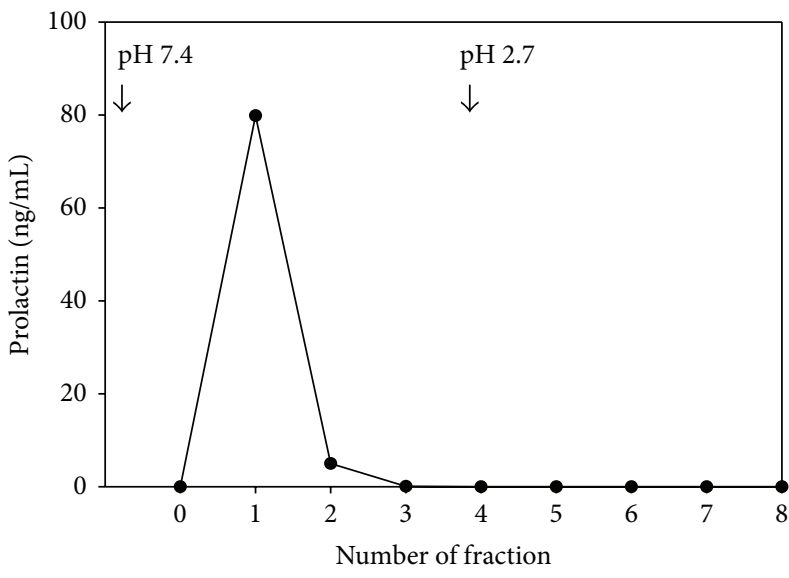

(a)

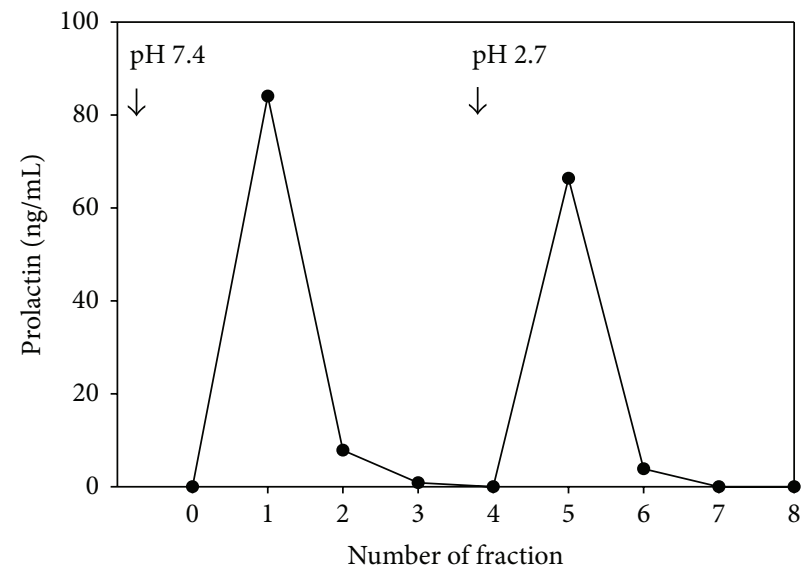

(b)

FIGURE 2: Representative affinity chromatography profiles of immunoreactive PRL in sera from one patient without anti-PRL autoantibody (a) and one patient whose test was positive for anti-PRL autoantibody (b) on a protein-G sepharose column ( $1 \mathrm{~mL})$. Samples $(1 \mathrm{~mL})$ were applied on the column, and fractions of $1 \mathrm{~mL}$ were collected. 
(10.5\%); in contrast, 81 of the 269 patients without MPRL had secondary causes of HPRL $(30.1 \%, P=0.004)$.

3.4. Follow-Up of Patients. Imaging investigations (computed tomography or magnetic resonance imaging) were performed in 16/57 (28.1\%) patients who had MPRL and in $36 / 269(13.4 \%)$ patients without MPRL. The frequency of abnormalities was exclusively found in patients without MPRL (11 were normal, 18 revealed a microadenoma, and 7 revealed a macroadenoma). All imaging studies were normal in patients with MPRL.

Forty-four (77.2\%) patients with MPRL and 147 (54.6\%) patients without MPRL were treated with dopamine agonists $(P=0.003)$. Symptomatic improvement occurred in 79 of 147 (53.7\%) patients without MPRL and in 4 of 44 (9.1\%) patients with MPRL $(P<0.001)$. A diagnosis of polycystic ovary syndrome was made in 22 of 269 (8.2\%) patients without MPRL and in 4 of $57(7.0 \%)$ patients with MPRL $(P=0.93)$.

3.5. Relationship between the Percentage of Serum PRL Precipitated by PEG and the Percentage of MPRL by Gel Filtration Chromatography. We observed a significant positive correlation between the percentage of serum PRL precipitated by PEG (PRL in serum-PRL in supernatant after PEG precipitation/PRL in serum $\times 100)$ and the percentage of MPRL by gel filtration chromatography (as gold standard to detect the presence of MPRL, $r=0.92, P<0.001$ ) (Figure 3). The area under the receiver operating characteristic curve was 0.998 (95\% CI $0.988-1, P<0.001$ ). The optimal cut-off point was $\geq 49.2 \%$; this cut-off yielded a sensitivity of $100 \%$ (95\% CI 93.7-100), specificity of 99.3\% (95\% CI 97.3-99.9), and positive and negative predictive values of $95.8 \%(95 \%$ CI 87.5-98.7) and 99.8\% (95\% CI 98.2-100), respectively. At this cut-off point, positive and negative likelihood ratios were 107.1 (95\% CI 31.2-367.8) and 0.009 (95\% CI 0.0010.14 ), respectively. As shown in Figure 1, there were two falsepositive samples; however, these serum samples displayed a variable pattern by gel filtration chromatography with a percentage of MPRL of 40.8 and $44.6 \%$, respectively.

\section{Discussion}

Several publications have associated asymptomatic HPRL with the predominance of MPRL [7, 18, 19, 23]. The frequency reported of MPRL in hyperprolactinemic subjects using similar methodologies has been 15-46\% [6-13]. However, the frequency of MPRL in hyperprolactinemic women with signs and symptoms related to elevated serum PRL levels is unknown. This study demonstrates that $17.5 \%$ of our selected hyperprolactinemic women studied (i.e., presenting with menstrual irregularities, galactorrhea, infertility, or alterations in libido) had significant MPRL. This finding is in accordance with a recently published study, which showed a frequency that is similar $(11.5 \%, 95 \%$ CI $6.9-16.1 \%)$ in a group of selected hyperprolactinemic patients with infertility [24]. Although the nature of MPRL is still unclear in some subjects with HPRL, the present study clearly indicates that the etiology of MPRL in the majority of patients is due to anti-PRL autoantibodies, mainly in those with very high

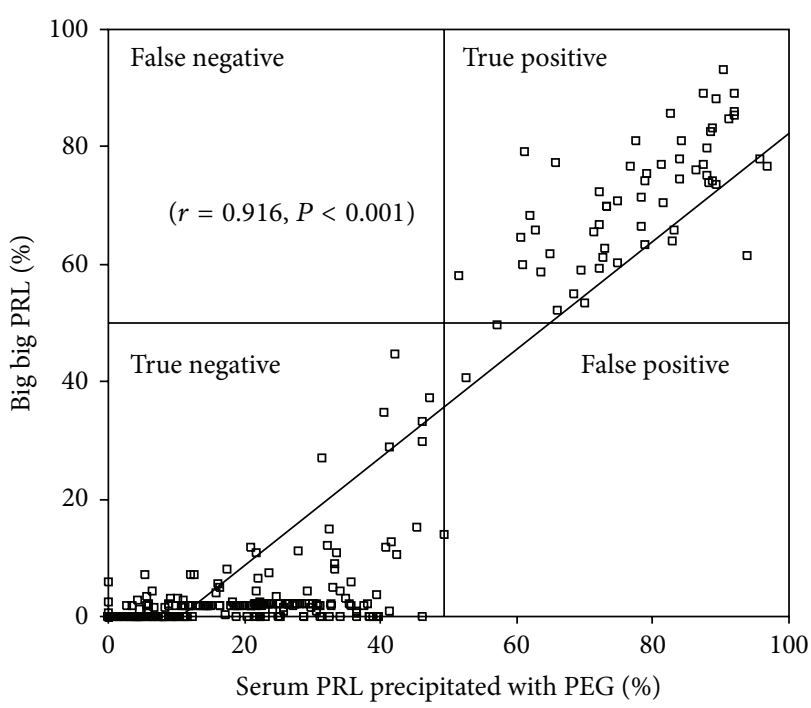

FIgURE 3: Relationship between the percentages of big big PRL (as determined by gel filtration chromatography) present in serum samples from 326 hyperprolactinemic patients (269 without macroprolactinemia and 57 with macroprolactinemia) and percentages of serum PRL precipitated by PEG. The horizontal line denotes the $50 \%$ limit for percentage of macroprolactinemia, and the vertical line is set to $49.2 \%$ of serum PRL precipitated by PEG (see Section 3).

serum PRL levels without a proven cause of the HPRL [4, 25, 26]. Other minor causes of MPRL have been attributed to formation of aggregates of monomeric PRL with different glycosylation degrees [4].

It has been suggested that the anti-PRL autoantibody itself is the cause of HPRL; to explain this, the following observations have been proposed as follows: (1) the PRL-antibody complex is eliminated more slowly from the bloodstream than free PRL $[16,27]$; (2) the PRL-IgG complex could block feedback mechanisms in the endocrine system, resulting in a low level of serum PRL to the hypothalamus and pituitary; this point may also be relevant in the presentation of active PRL to cells bearing PRL receptors [22]; and (3) there exist a positive correlation between the proportion of PRL bound to IgG and serum PRL levels ([3, 22, 27], as in the present study).

Measurements of serum immunoreactive PRL concentrations in hyperprolactinemic subjects do not always correlate with the clinical findings. In fact, several authors have reported that asymptomatic HPRL is frequently associated with the presence of molecular heterogeneity, particularly the predominant presence of MPRL [8-10, 18, 28]. However, other authors have reported that patients with MPRL cannot be distinguished from patients with HPRL, but without MPRL on the basis of clinical features alone $[12,13]$. These discrepancies may be due to population studied (as our patients included), the use of different immunoassays, or criteria for definition of MPRL. In this study, although the frequency of galactorrhea was significantly higher in patients without MPRL than in patients with MPRL, these differences are not sufficient to distinguish between these two entities. Moreover, since signs and symptoms of HPRL are nonspecific 
and relatively common, it is possible that some patients with MPRL experience these signs and symptoms coincidentally, but unrelated to PRL, resulting in misdiagnosis and inappropriate investigation and treatment as reported in this study and by prior studies $[7,10,11]$. Indeed, there are reports of patients who underwent pituitary surgical exploration [29, 30]. Moreover, the findings that serum free PRL (monomeric PRL) levels from patients with MPRL were $\leq 22.2 \mathrm{ng} / \mathrm{mL}$ and that all images of studies were normal, as well as the fact that the symptomatic improvement after the treatment with dopamine agonists occurred in only $9.1 \%$ of patients suggest that signs and symptoms experienced by our patients studied were coincidental rather than being attributable to a true HPRL.

Abnormal findings on pituitary imaging were more frequent in patients without MPRL than in patients with MPRL (69.4 and 0\%, resp.), and these results are consistent with those of other studies $[8,10,13]$. On the other hand, the finding that the treatment with dopamine agonists was prescribed in $77.2 \%$ of patients with MPRL is consistent with previous reports ranging from 76.5 to $86.7 \%[7,10]$.

As being consistent with previous studies [31, 32], the results of this study indicate that precipitation of MPRL by PEG is an effective technique for detecting MPRL. The percentage of MPRL with values of $\geq 49.2 \%$ precipitated with PEG (PRL recovery of $\leq 50.7 \%$ ) reflected an ideal cut-off point for detecting exclusive predominant pattern of MPRL by gel filtration chromatography (as gold standard) with high accuracy. Furthermore, values below $49.2 \%$ of precipitation of serum PRL can be interpreted as virtually excluding MPRL and solely the presence of the monomeric PRL form circulating in the blood. The least common cases with precipitation between $49.2 \%$ and lower than $60.0 \%$ require gel filtration chromatography to characterize the predominant molecular form of PRL. In this vein, another definition of MPRL has been proposed requiring that concentrations of free PRL fall in the range of serum samples from normoprolactinemic healthy subjects treated with PEG $[10,11]$. This approach is reasonable because it can identify patients who need further investigations and treatments for HPRL.

In accordance to previous studies $[10,11,13,33]$, the present results provide more support to the notion that MPRL can be a benign variant and that treatment with dopamine agonists, imaging investigations, or prolonged follow-up is not necessary. However, the patients with MPRL and menstrual irregularities, galactorrhea, infertility, or alteration in libido should be investigated for other causes different than HPRL.

In summary, we have demonstrated that macroprolactinemia is a prevalent cause of HPRL among women presenting with menstrual irregularities, galactorrhea, infertility, or alterations in libido attended by gynecology and endocrinology clinic, and we confirmed that the main etiology of MPRL is due to presence of anti-PRL autoantibodies. Macroprolactinemia should be taken into account as a probable cause of HPRL in order to avoid misdiagnosis and unnecessary investigations and treatment. The percentage of serum PRL precipitated by PEG provides a good estimation of the predominant presence of big big PRL as determined by gel filtration chromatography. Nevertheless, the diagnostic performance of the percentage of serum PRL precipitated by PEG should be validated locally by each laboratory because of the variability in laboratory methods used to measure PRL and the criteria to establish cut-off points.

\section{Conflict of Interests}

There is no conflict of interests that would prejudice the impartiality of this scientific work.

\section{Acknowledgment}

This study was supported by a grant from the FIS/IMSS/ PROT/G09/750, México, to Alfredo Leaños-Miranda.

\section{References}

[1] I. S. Fraser and Z. G. Lun, "Polymers of prolactin and their clinical significance," Obstetrical and Gynecological Survey, vol. 45, no. 8, pp. 515-520, 1990.

[2] V. Leite, H. Cosby, L. G. Sobrinho, A. Fresnoza, M. A. Santos, and H. G. Friesen, "Characterization of big, big prolactin in patients with hyperprolactinaemia," Clinical Endocrinology, vol. 37, no. 4, pp. 365-372, 1992.

[3] N. Hattori, K. Ikekubo, T. Ishihara, K. Moridera, M. Hino, and H. Kurahachi, "Correlation of the antibody titers with serum prolactin levels and their clinical course in patients with antiprolactin autoantibody," European Journal of Endocrinology, vol. 130, no. 5, pp. 438-445, 1994.

[4] J. de Schepper, J. Schiettecatte, B. Velkeniers et al., "Clinical and biological characterization of macroprolactinemia with and without prolactin-IgG complexes," European Journal of Endocrinology, vol. 149, no. 3, pp. 201-207, 2003.

[5] A. Leaños-Miranda, G. Cárdenas-Mondragón, R. RiveraLeaños, A. Ulloa-Aguirre, and V. Goffin, "Application of new homologous in vitro bioassays for human lactogens to assess the actual bioactivity of human prolactin isoforms in hyperprolactinaemic patients," Clinical Endocrinology, vol. 65, no. 2, pp. 146-153, 2006.

[6] M. N. Fahie-Wilson and S. G. Soule, "Macroprolactinaemia: contribution to hyperprolactinaemia in a district general hospital and evaluation of a screening test based on precipitation with polyethylene glycol," Annals of Clinical Biochemistry, vol. 34, no. 3, pp. 252-258, 1997.

[7] A. O. Olukoga and J. W. Kane, "Macroprolactinaemia: validation and application of the polyethylene glycol precipitation test and clinical characterization of the condition," Clinical Endocrinology, vol. 51, no. 1, pp. 119-126, 1999.

[8] O. M. Hauache, A. J. Rocha, A. C. M. Maia Jr., R. M. B. Maciel, and J. G. H. Vieira, "Screening for macroprolactinaemia and pituitary imaging studies," Clinical Endocrinology, vol. 57, no. 3, pp. 327-331, 2002.

[9] S. Vallette-Kasic, I. Morange-Ramos, A. Selim et al., "Macroprolactinemia revisited: a study on 106 patients," Journal of Clinical Endocrinology and Metabolism, vol. 87, no. 2, pp. 581-588, 2002.

[10] A. M. Suliman, T. P. Smith, J. Gibney, and T. J. McKenna, "Frequent misdiagnosis and mismanagement of hyperprolactinemic patients before the introduction of macroprolactin screening: application of a new strict laboratory definition of 
macroprolactinemia," Clinical Chemistry, vol. 49, no. 9, pp. 1504-1509, 2003.

[11] J. Gibney, T. P. Smith, and T. J. McKenna, "The impact on clinical practice of routine screening for macroprolactin," Journal of Clinical Endocrinology and Metabolism, vol. 90, no. 7, pp. 39273932, 2005.

[12] C. R. McCudden, J. L. Sharpless, and D. G. Grenache, "Comparison of multiple methods for identification of hyperprolactinemia in the presence of macroprolactin," Clinica Chimica Acta, vol. 411, no. 3-4, pp. 155-160, 2010.

[13] S. Isik, D. Berker, Y. A. Tutuncu et al., "Clinical and radiological findings in macroprolactinemia," Endocrine, vol. 41, pp. 327333, 2012.

[14] A. Leaños, D. Pascoe, A. Fraga, and F. Blanco-Favela, "Anti-prolactin autoantibodies in systemic lupus erythematosus patients with associated hyperprolactinemia," Lupus, vol. 7, no. 6, pp. 398-403, 1998.

[15] D. Pascoe-Lira, G. Duran-Reyes, I. Contreras-Hernández, L. Manuel-Apolinar, F. Blanco-Favela, and A. Leaños-Miranda, "Frequency of macroprolactinemia due to autoantibodies against prolactin in pregnant women," Journal of Clinical Endocrinology and Metabolism, vol. 86, no. 2, pp. 924-929, 2001.

[16] A. Leaños-Miranda, K. A. Chávez-Rueda, and F. Blanco-Favela, "Biologic activity and plasma clearance of prolactin-IgG complex in patients with systemic lupus erythematosus," Arthritis and Rheumatism, vol. 44, no. 4, pp. 866-875, 2001.

[17] A. Glezer, C. R. J. Soares, J. G. Vieira et al., "Human macroprolactin displays low biological activity via its homologous receptor in a new sensitive bioassay," Journal of Clinical Endocrinology and Metabolism, vol. 91, no. 3, pp. 1048-1055, 2006.

[18] R. D. Jackson, J. Wortsman, and W. B. Malarkey, "Characterization of a large molecular weight prolactin in women with idiopathic hyperprolactinemia and normal menses," Journal of Clinical Endocrinology and Metabolism, vol. 61, no. 2, pp. 258264, 1985.

[19] A. Alfonso, K. I. Rieniets, and R. A. Vigersky, "Incidence and clinical significance of elevated macroprolactin levels in patients with hyperprolactinemia," Endocrine Practice, vol. 12, no. 3, pp. 275-280, 2006.

[20] T. P. Smith, L. Kavanagh, M. Healy, and T. J. McKenna, "Technology insight: measuring prolactin in clinical samples," Nature Clinical Practice Endocrinology and Metabolism, vol. 3, no. 3, pp. 279-289, 2007.

[21] A. Leaños-Miranda and I. Contreras-Hernández, "Antiprolactin autoantibodies are associated with hyperprolactinemic status in men infected with human immunodeficiency virus," Endocrine, vol. 19, no. 2, pp. 139-146, 2002.

[22] A. Leaños-Miranda, D. Pascoe-Lira, K. A. Chávez-Rueda, and F. Blanco-Favela, "Detection of macroprolactinemia with the polyethylene glycol precipitation test in systemic lupus erythematosus patients with hyperprolactinemia," Lupus, vol. 10, no. 5, pp. 340-345, 2001.

[23] I. S. Fraser, Z. G. Lun, J. P. Zhou et al., "Detailed assessment of big big prolactin in women with hyperprolactinemia and normal ovarian function," Journal of Clinical Endocrinology and Metabolism, vol. 69, no. 3, pp. 585-592, 1989.

[24] K. Thirunavakkarasu, P. Dutta, S. Sridhar et al., "Macroprolactinemia in hyperprolactinemic infertile women," Endocrine, 2013.

[25] N. Hattori, T. Ishihara, K. Ikekubo, K. Moridera, M. Hino, and H. Kurahachi, "Autoantibody to human prolactin in patients with idiopathic hyperprolactinemia," Journal of Clinical Endocrinology and Metabolism, vol. 75, no. 5, pp. 1226-1229, 1992.

[26] P. Amadori, C. Dilberis, A. Marcolla, M. Pinamonti, P. Menapace, and A. Valentini, "Identification of IgG-immunocomplex macroprolactin with an immunometric "sandwich" system: technical and clinical considerations," Journal of Endocrinological Investigation, vol. 27, no. 11, pp. 1022-1028, 2004.

[27] N. Hattori and C. Inagaki, "Anti-prolactin (PRL) autoantibodies cause asymptomatic hyperprolactinemia: bioassay and clearance studies of PRL-immunoglobulin G complex," Journal of Clinical Endocrinology and Metabolism, vol. 82, no. 9, pp. 31073110, 1997.

[28] H. Leslie, C. H. Courtney, P. M. Bell et al., "Laboratory and clinical experience in 55 patients with macroprolactinemia identified by a simple polyethylene glycol precipitation method," Journal of Clinical Endocrinology and Metabolism, vol. 86, no. 6, pp. 2743-2746, 2001.

[29] F. A. Cattaneo and M. N. Fahie-Wilson, "Concomitant occurrence of macroprolactin, exercise-induced amenorrhea, and a pituitary lesion: a diagnostic pitfall. Case report," Journal of Neurosurgery, vol. 95, no. 2, pp. 334-337, 2001.

[30] A. O. Olukoga, T. L. Dornan, and J. W. Kane, "Three cases of macroprolactinaemia," Journal of the Royal Society of Medicine, vol. 92, no. 7, pp. 342-344, 1999.

[31] M. N. Fahie-Wilson, "Polyethylene glycol precipitation as a screening method for macroprolactinemia," Clinical Chemistry, vol. 45, no. 3, pp. 436-437, 1999.

[32] J. G. H. Vieira, T. T. Tachibana, L. H. Obara, and R. M. B. Maciel, "Extensive experience and validation of polyethylene glycol precipitation as a screening method for macroprolactinemia," Clinical Chemistry, vol. 44, no. 8 I, pp. 1758-1759, 1998.

[33] I. R. Wallace, N. Satti, C. H. Courtney et al., "Ten-year clinical follow-up of a cohort of 51 patients with macroprolactinemia establishes it as a benign variant," Journal of Clinical Endocrinology and Metabolism, vol. 95, no. 7, pp. 3268-3271, 2010. 


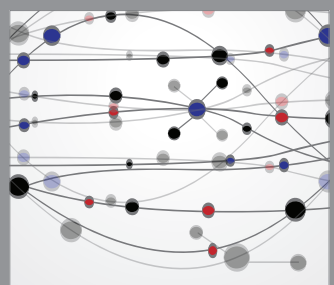

The Scientific World Journal
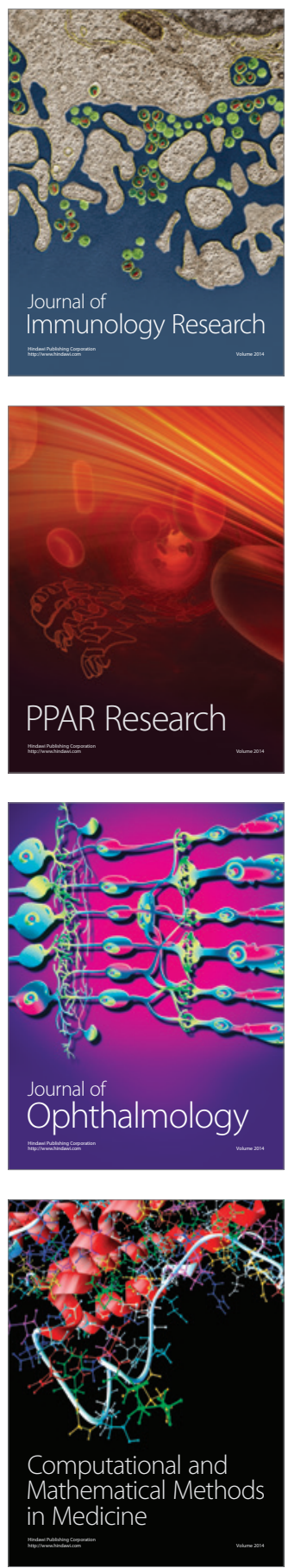

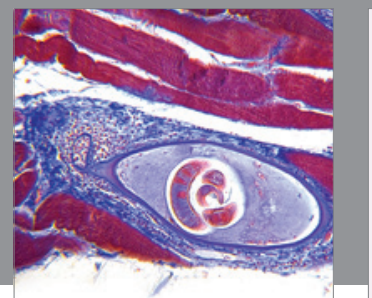

Gastroenterology

Research and Practice
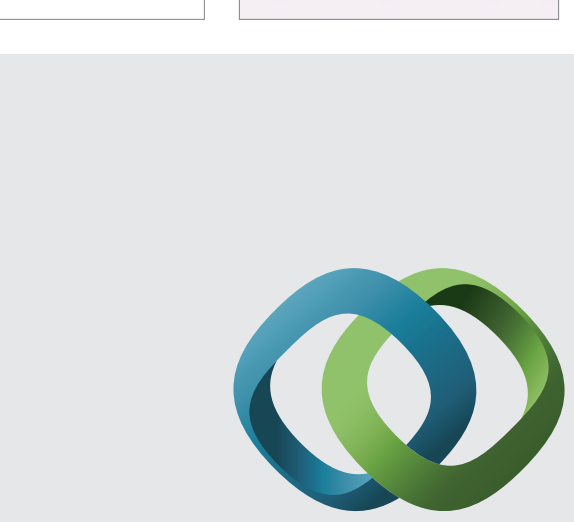

\section{Hindawi}

Submit your manuscripts at

http://www.hindawi.com
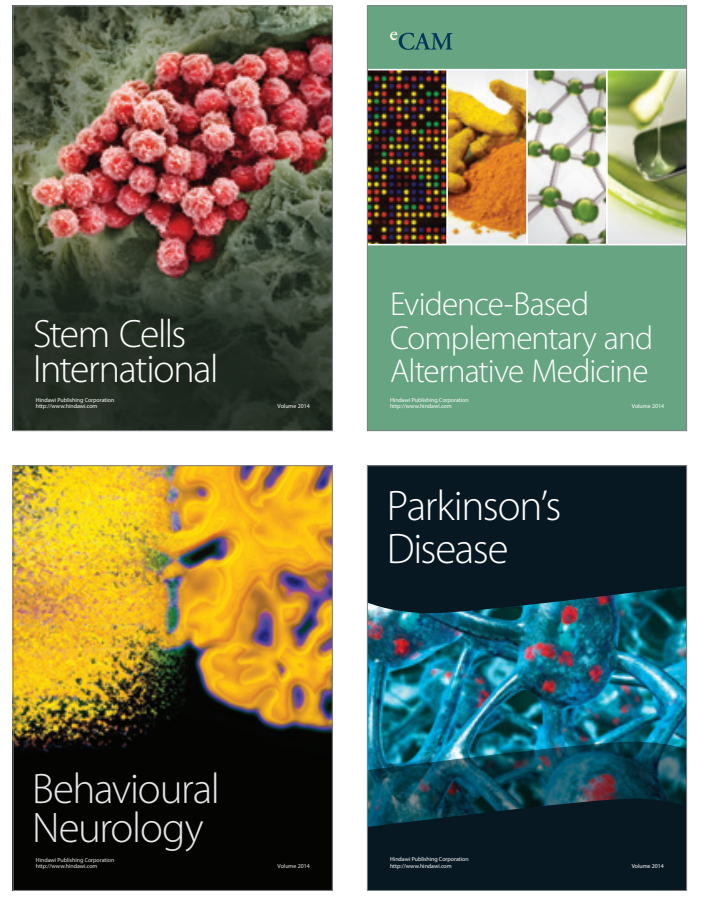
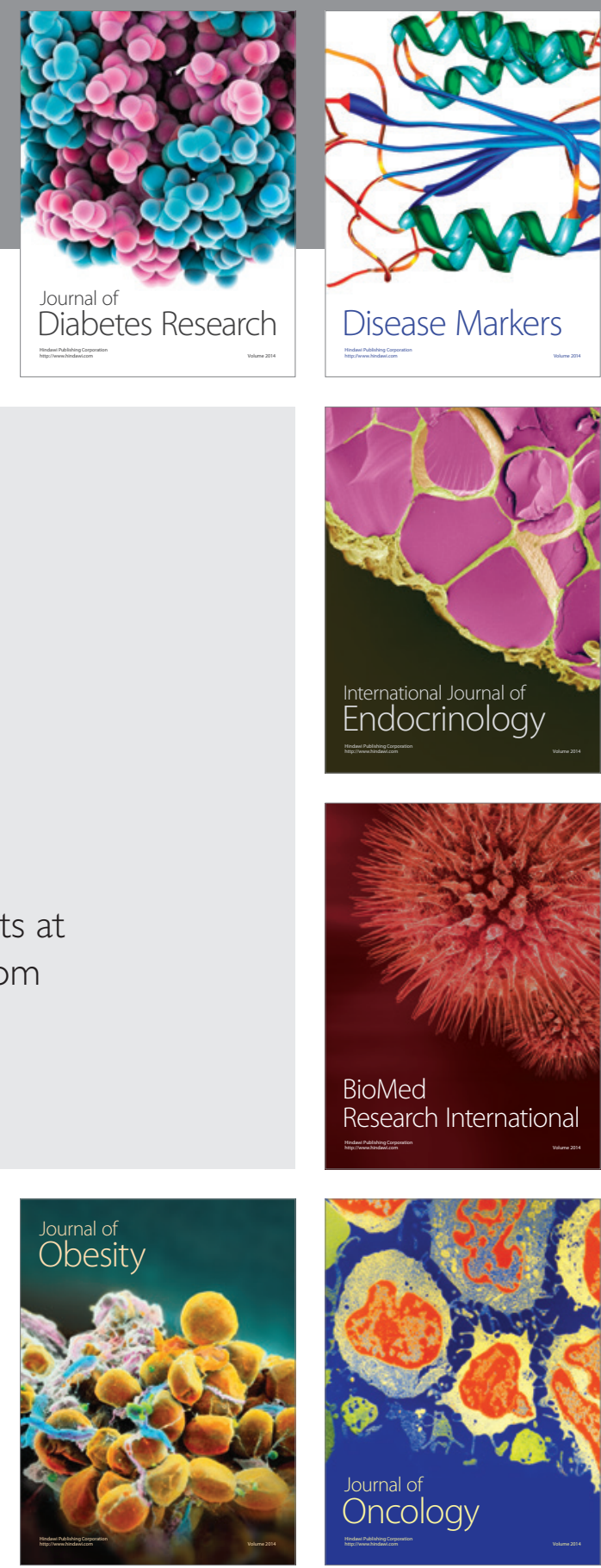

Disease Markers
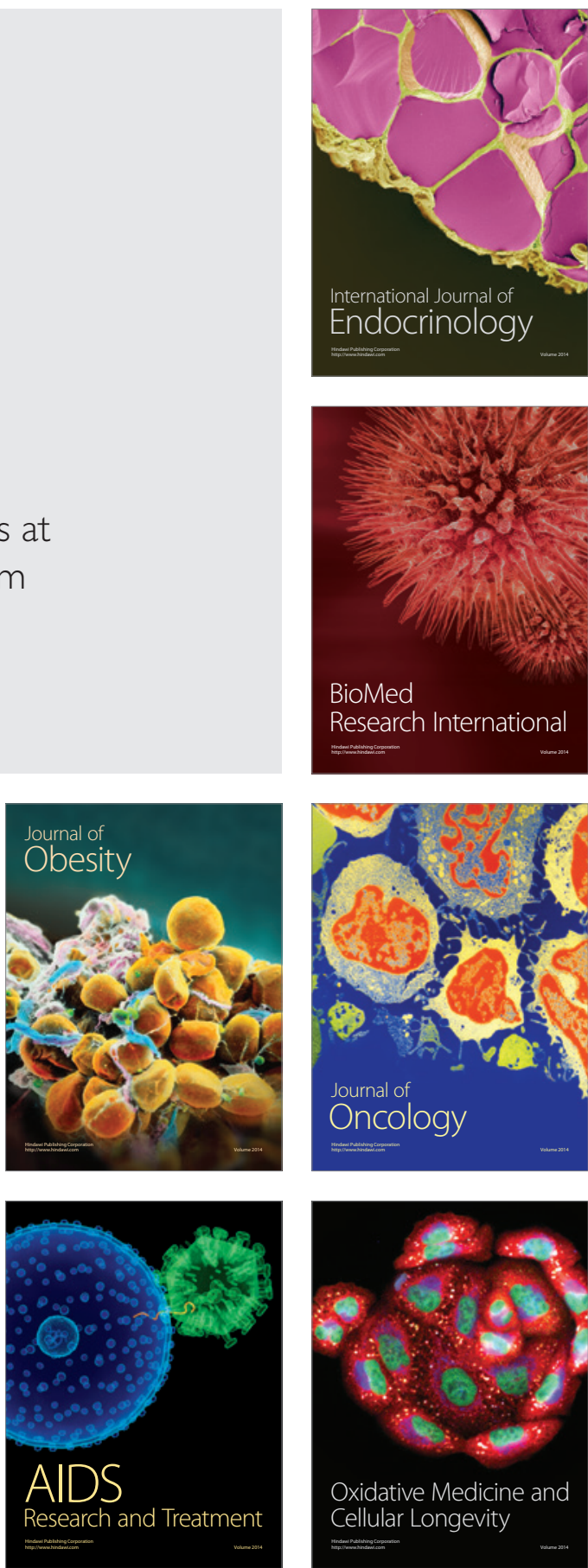